\title{
Fragment-based development of bacterial DsbA inhibitors as novel anti-virulence agents
}

\section{Geqing Wang ${ }^{1}$, Wesam Alwan², Matthew Bentley², Biswaranjan Mohanty ${ }^{2}$, Bradley Doak ${ }^{2}$, Rabeb Dhouib ${ }^{3}$, Makrina Totsika ${ }^{3}$, Róisín McMahon ${ }^{4}$, Benvenuto Capuano ${ }^{2}$, Peter Scammells ${ }^{2}$, Jennifer Martin ${ }^{4}$, Martin Scanlon ${ }^{2}$, Begoña Heras ${ }^{1}$}

\author{
${ }^{l}$ Department of Biochemistry and Genetics, La Trobe Institute for Molecular Science, La Trobe University, \\ Bundoora, VIC, 3086, Australia \\ ${ }_{2}^{2}$ Medicinal Chemistry, Monash Institute of Pharmaceutical Sciences, Monash University, Parkville, VIC, 3052, Australia \\ ${ }^{3}$ Institute of Health and Biomedical Innovation, Queensland University of Technology, Kelvin Grove, QLD, 4059 \\ ${ }^{4}$ Griffith Institute for Drug Discovery, Griffith University, Nathan, QLD, 4111, Australia
}

g.wang@latrobe.edu.au,b.heras@latrobe.edu.au

\begin{abstract}
Antibiotic resistance is growing to dangerously high levels and poses a serious threat to global public health. The emergence and spread of resistance mechanisms to all antibiotics introduced into the clinic jeopardize the effectiveness of current treatments. Traditionally, antibiotics have been designed to inhibit bacterial viability or impair their growth; these mechanisms induce a strong selection pressure for resistance development. To overcome this problem, an alternate approach is to disarm bacterial virulence without killing them, which potentially reduces selection pressure and delays the emergence of resistance.

In this project, we target the thiol-disulfide oxidoreductase enzyme DsbA which catalyzes disulfide bond formation in the periplasm of Gram-negative bacteria. DsbA facilitates folding of multiple virulent factors and acts as a major regulator of bacterial virulence. Bacteria lacking a functional DsbA display reduced virulence, increased sensitivity to antibiotics and diminished capacity to cause infection in many Gram-negative pathogens [1]. We carried out a fragment screening campaign against Escherichia coli DsbA and identified the first small molecule inhibitors that bind to the catalytic site of DsbA and inhibit DsbA activity in vitro and cell-based assays $[2,3,4]$. By exploiting an array of biophysical/biochemical tools (NMR, SPR, X-ray crystallography and in vitro assays), we aim to optimize these DsbA inhibitors from fragment hits to high-affinity leads. Herein we report our established drug discovery pipeline and current efforts in developing DsbA inhibitors. The goal of this work is to develop a new generation of antimicrobials with a novel mode of action that could be used alone or in combination with existing drugs to treat multi-drug resistant infections.
\end{abstract}

[1] B. Heras, et al. (2009), DSB proteins and bacterial pathogenicity, Nat. Rev. Microbiol., 7,215-225

[2] Duncan L.F., et al. (2019), Molecules 24 (20)

[3] L.A. Adams, et al. (2015), Application of fragment-based screening to the design of inhibitors of Escherichia coli DsbA, Angew. Chem. Int. Ed., $54,2179-2184$

[4] M.Totsika, et al. (2018), Inhibition of diverse DsbA enzymes in multi-DsbA encoding pathogens. Antioxid. Redox. Signal., 29,7

Keywords: Oxireductase; fragment-based drug discovery; antibacterials; drug resistance; X-ray crystallography; protein dynamics 